CERN-TH.6549/92

\title{
Feynman rules for fermion-number-violating interactions
}

\author{
A. DENNER \\ CERN, Geneva, Switzerland \\ H. ECK, O. HAhN, J. KÜBLBECK \\ Physikalisches Institut, University of Würzburg, Germany
}

\begin{abstract}
:
We present simple algorithmic Feynman rules for fermion-number-violating interactions. They do not involve explicit charge-conjugation matrices and resemble closely the familiar rules for Dirac fermions. We insist on a fermion flow through the graphs along fermion lines and get the correct relative signs between different interfering Feynman graphs as in the case of Dirac fermions. We only need the familiar Dirac propagator and less vertices than in the usual treatment of fermion-number-violating interactions.
\end{abstract}

CERN-TH.6549/92

June 1992 


\section{Introduction}

Besides the electroweak standard model, its supersymmetric extension has gained a lot of interest. There already exist a large number of tree-level calculations within the supersymmetric standard model and recently the first one-loop corrections have been evaluated. In contrast with the electroweak standard model, supersymmetric theories involve interactions that violate the fermion number.

For such theories, there exist up to now no convincing Feynman rules that treat fermions in a simple and unique way. The problem becomes most evident for Majorana fermions. Their self-conjugacy allows for a variety of different contractions, which acquire different signs originating from the anticommutativity of fermionic operators. As a consequence the Relative Sign of Interfering Feynman graphs (RSIF) has to be determined very carefully. Therefore many authors prefer to use the Wick theorem for practical calculations in particular for the determination of the RSIF.

Feynman rules for Majorana fermions were given in Refs. [1, 2]. They involve vertices and propagators with clashing arrows. This is reflected by the appearance of the chargeconjugation matrix in the Feynman rules for vertices and propagators. All Feynman graphs are constructed as usual from the available couplings. Majorana propagators are assigned arbitrary directions. Applying the Feynman rules for vertices and propagators, one ends up with Dirac chains involving Dirac matrices, transposed Dirac matrices, and charge-conjugation matrices. In this approach the RSIF cannot be read off the graphs, but has to be determined independently from the Wick contractions. We refer to this as the conventional Feynman rules for Majorana fermions.

In Ref. [3] a first simplification of the Feynman rules for Majorana fermions has been achieved. The number of propagators and vertices has been reduced, and only the vertices without charge-conjugation matrix and the conventional fermion propagator are left. In addition, a recipe for the determination of the RSIF has been given. However, this recipe seems ad hoc and non-transparent and it is not immediately evident that it yields the correct signs in all cases.

The conventional method to find the RSIF by determining the permutation of the external fermions was used in a recent work [4]. There the vertices are given only with external spinors from which the propagators have to be constructed. To obtain the usual analytic expression in terms of propagators and vertices, several algebraic manipulations have to be performed. This seems to be unnecessarily complicated and pretty far away from the familiar way of translating Feynman graphs into analytical expressions, with Feynman rules for propagators and vertices.

We suggest Feynman rules which are very close to the rules for Dirac fermions, but are valid for all theories with fermion-number violation, in particular for Majorana fermions. We follow the idea described in Ref. [3] of maintaining a continuous fermion flow. This forces one to introduce two analytical expressions for each vertex, one for fermion flow parallel and one for fermion flow antiparallel to the flow of fermion number. However, keeping track of the anticommutativity of fermionic operators, we derive Feynman rules for the reversed fermion flow which have signs different from those given in Ref. [3]. As a consequence we only need the well-known propagators for Dirac fermions, we need less vertices than in the conventional treatment, and the RSIF is determined from the permu- 
tation of the external fermions as usual. Moreover, our Feynman rules naturally apply to theories containing explicit charge-conjugate fermion fields, which were not considered in Refs. [3, 4].

Our set of rules is strictly algorithmic and can easily be implemented into a symbolic Feynman graph and amplitude generator. In fact it has been developed in order to incorporate the supersymmetric standard model into the program FeynArts [5].

The paper is organized as follows: In section 2 we introduce a generic Lagrangian with Majorana and Dirac fermions. Based on the Wick contraction formalism we review the origin of the ordinary Feynman rules for Dirac fermions and generalize these to processes involving Majorana fields. In section 3 we illustrate our procedure by giving four examples and compare them with earlier methods. The generalization to theories containing explicit charge-conjugate fermion fields is presented in section 4 .

\section{Feynman rules for general fermionic interactions}

\subsection{Lagrangian}

We consider the generic Lagrangian $\mathcal{L}$ for Majorana fields $\lambda$ and Dirac fields $\psi$ as given in Ref. [2] augmented by a pure Dirac interaction term:

$$
\begin{aligned}
\mathcal{L}= & \frac{1}{2} \bar{\lambda}_{a}\left(i \not \partial-M_{a}\right) \lambda_{a}+\bar{\psi}_{a}\left(i \not \partial-m_{a}\right) \psi_{a} \\
& +\frac{1}{2} g_{a b c}^{i} \bar{\lambda}_{a} \Gamma_{i} \lambda_{b} \Phi_{c}+k_{a b c}^{i} \bar{\lambda}_{a} \Gamma_{i} \psi_{b} \Phi_{c}^{*}+k_{a b c}^{i *} \bar{\psi}_{b} \Gamma_{i} \lambda_{a} \Phi_{c}+h_{a b c}^{i} \bar{\psi}_{a} \Gamma_{i} \psi_{b} \Phi_{c}
\end{aligned}
$$

where $\Gamma_{i}=1, i \gamma_{5}, \gamma_{\mu} \gamma_{5}, \gamma_{\mu}, \sigma_{\mu \nu}$ and $g_{a b c}^{i}, k_{a b c}^{i}$ and $h_{a b c}^{i}$ are coupling constants. The field $\Phi$ summarizes scalar and vector fields. We suppress non-fermionic contributions as these are treated as usual.

In order to introduce our Feynman rules, we derive the analytic expression for a certain Feynman graph with fermionic interactions ${ }^{1}$. To this Feynman graph uniquely correspond certain equivalent sets of contractions of the matrix element

$$
\left\langle 0\left|b_{i_{1}} \ldots d_{i_{m}} T[(\bar{\chi} \Gamma \chi) \ldots(\bar{\chi} \Gamma \chi)] b_{i_{m+1}}^{\dagger} \ldots d_{i_{n}}^{\dagger}\right| 0\right\rangle .
$$

Here $b_{i}^{\dagger}, d_{i}^{\dagger}$ are the creation operators of fermions and antifermions, respectively, and $b_{i}, d_{i}$ the corresponding annihilation operators. The index $i$ summarizes momentum $p_{i}$, spin $s_{i}$ and possibly further quantum numbers. The field $\chi$ stands for both Majorana and Dirac fields, and $\Gamma$ denotes a generic fermionic interaction including Dirac matrices, coupling constants and the boson fields, e.g.

$$
\bar{\psi} \Gamma \psi=h_{a b c}^{i} \bar{\psi}_{a} \Gamma_{i} \psi_{b} \Phi_{c}
$$

for Dirac fermions. Our aim is to reorder the operators (2.2) for a given set of contractions such that the Feynman rules can be read off directly. In particular, we keep track of the signs resulting from the anticommutativity of the fermion fields: interchanging two single fermionic operators yields a minus sign, reordering of the interaction Lagrangians does not.

\footnotetext{
${ }^{1}$ We adopt the conventions of $[6]$.
} 


\subsection{Dirac fields}

We first review the derivation for usual Dirac fermions. By reordering the interaction Lagrangians and the annihilation and creation operators of the external particles, each contribution to the matrix element (2.2) can be brought into the form

$$
\begin{aligned}
(-1)^{P} & b_{i_{1}}(\bar{\psi} \Gamma \psi) \ldots \ldots(\bar{\psi} \Gamma \psi)(\bar{\psi} \Gamma \psi) b_{i_{k}}^{\dagger} \ldots b_{i_{l}}(\bar{\psi} \Gamma \psi) \ldots \ldots(\bar{\psi} \Gamma \psi)(\bar{\psi} \Gamma \psi) d_{i_{m}} \ldots \\
& d_{i_{n}}^{\dagger}(\bar{\psi} \Gamma \psi) \ldots \ldots(\bar{\psi} \Gamma \psi)(\bar{\psi} \Gamma \psi) b_{i_{o}}^{\dagger} \ldots d_{i_{p}}^{\dagger}(\bar{\psi} \Gamma \psi) \ldots . .(\bar{\psi} \Gamma \psi)(\bar{\psi} \Gamma \psi) d_{i_{q}} \ldots \\
& (\bar{\psi} \Gamma \psi) \ldots \ldots .(\bar{\psi} \Gamma \psi)(\bar{\psi} \Gamma \psi),
\end{aligned}
$$

where the brackets denote the contractions. The signs emerging from this reordering are only due to the permutation of the annihilation and creation operators among themselves. This causes the factor $(-1)^{P}$, where $P$ is the parity of this permutation. We have written down one example for each type of open fermion line and for a closed fermion loop. The two operators of a contraction are adjacent to each other, apart from one contraction in every closed fermion loop. The individual contractions yield the familiar analytical expressions in momentum space. With the explicit expressions of the free spinor fields

$$
\begin{aligned}
& \psi(x)=\int \frac{d^{3} k}{(2 \pi)^{3} 2 E} \sum_{s}\left[b(k, s) u(k, s) e^{-i k x}+d^{\dagger}(k, s) v(k, s) e^{i k x}\right] \\
& \bar{\psi}(x)=\int \frac{d^{3} k}{(2 \pi)^{3} 2 E} \sum_{s}\left[b^{\dagger}(k, s) \bar{u}(k, s) e^{i k x}+d(k, s) \bar{v}(k, s) e^{-i k x}\right]
\end{aligned}
$$

the internal propagator reads

$$
\psi \bar{\psi}=\langle 0|T(\psi \bar{\psi})| 0\rangle \quad \longrightarrow \quad \frac{1}{\not p-m}=S(p)
$$

where the propagating field $\psi$ carries a momentum $p$. The contractions with the external operators are obtained as

$$
\begin{array}{llll}
\stackrel{\psi}{\psi b_{i}^{\dagger}}=\left\langle 0\left|\psi(x) b^{\dagger}\left(p_{i}, s_{i}\right)\right| 0\right\rangle & \longrightarrow & u\left(p_{i}, s_{i}\right) \\
b_{i} \bar{\psi} & =\left\langle 0\left|b\left(p_{i}, s_{i}\right) \bar{\psi}(x)\right| 0\right\rangle \quad \longrightarrow & \bar{u}\left(p_{i}, s_{i}\right) \\
\bar{\psi} d_{i}^{\dagger} & =\left\langle 0\left|\bar{\psi}(x) d^{\dagger}\left(p_{i}, s_{i}\right)\right| 0\right\rangle & \longrightarrow & \bar{v}\left(p_{i}, s_{i}\right) \\
\square & & & \\
d_{i} \psi & =\left\langle 0\left|d\left(p_{i}, s_{i}\right) \psi(x)\right| 0\right\rangle & \longrightarrow & v\left(p_{i}, s_{i}\right) .
\end{array}
$$

In order to apply (2.6) and (2.7), we have to exchange the operators of the contractions $\psi d, d^{\dagger} \bar{\psi}$ as well as the first and the last field operator of the closed fermion chain in (2.4), leading to an additional minus sign for each of these contractions.

Using (2.6) and (2.7) the analytic expression in momentum space is obtained directly from (2.4) as products and traces of coupling and propagator matrices and spinors. Their order in the multiplication is naturally given as opposite to the fermion number flow. 
The RSIF is easily determined as follows: Each contributing graph gets the sign $(-1)^{P+L+V}$, where

(1) $P$ is the parity of the permutation of external spinors with respect to some reference order,

(2) $L$ is the number of closed fermion loops, and

(3) $V$ is the number of spinors $v$ and $\bar{v}$.

The factor $(-1)^{V}$ is universal for all graphs of a process and can therefore be omitted consistently.

\subsection{General fermion fields}

If we allow self-conjugate fields the contractions $\chi \chi$ and $\bar{\chi} \bar{\chi}$ are non-vanishing and the continuous fermion number flow is in general no longer maintained. Nevertheless we can reorder the interaction Lagrangians, and annihilation and creation operators for any set of contractions in (2.2) such that the fields which are contracted are in adjacent interaction Lagrangians. But now the fields which are contracted in general do not stand next to each other, as it is guaranteed in the case of Dirac fermions, because of the continuous fermion number flow. For instance, we can have the following contraction

$$
\ldots(\bar{\chi} \Gamma \chi) \ldots .
$$

It is, however, possible to reverse the interaction Lagrangian $\bar{\chi} \Gamma \chi$ by introducing the charge-conjugate fields

$$
\tilde{\chi}=C \bar{\chi}^{T}, \quad \bar{\chi}=-\chi^{T} C^{-1} .
$$

The charge-conjugation matrix $C$ fulfils $^{2}$

$$
C^{\dagger}=C^{-1}, \quad C^{T}=-C, \quad C \Gamma_{i}^{T} C^{-1}=\eta_{i} \Gamma_{i}
$$

(no summation over $i$ ), with

$$
\eta_{i}=\left\{\begin{aligned}
1 \text { for } \Gamma_{i}=1, i \gamma_{5}, \gamma_{\mu} \gamma_{5} \\
-1 \text { for } \Gamma_{i}=\gamma_{\mu}, \sigma_{\mu \nu}
\end{aligned}\right.
$$

From (2.9) and (2.10) we obtain

$$
\begin{aligned}
\bar{\chi} \Gamma \chi & =g_{a b c}^{i} \Phi_{c} \bar{\chi}_{a} \Gamma_{i} \chi_{b}=g_{a b c}^{i} \Phi_{c}\left(\bar{\chi}_{a} \Gamma_{i} \chi_{b}\right)^{T}=(-1) g_{a b c}^{i} \Phi_{c} \chi_{b}^{T} \Gamma_{i}^{T} \bar{\chi}_{a}^{T} \\
& =g_{a b c}^{i} \Phi_{c} \overline{\widetilde{\chi}_{b}} C \Gamma_{i}^{T} C^{-1} \widetilde{\chi}_{a}=g_{a b c}^{i} \Phi_{c} \overline{\widetilde{\chi}_{b}} \eta_{i} \Gamma_{i} \widetilde{\chi}_{a}=: \overline{\widetilde{\chi}} \Gamma^{\prime} \tilde{\chi}
\end{aligned}
$$

with

$$
\Gamma^{\prime}=C \Gamma^{T} C^{-1}
$$

\footnotetext{
${ }^{2} C$ has been chosen such that $\widetilde{(\widetilde{\chi})}=\chi$.
} 
In (2.12) $g$ stands for any of the coupling constants $g, k$ or $h$. Note that the factor $(-1)$ originating from the anticommutation of the fermion fields in the fourth expression of (2.12) has been incorporated into the definition of $\Gamma^{\prime}$. For Majorana fermions, $\tilde{\chi}=\chi$. Therefore, if both $\chi^{\text {'s }}$ are Majorana fermions (2.12) implies $\eta_{i} g_{a b c}^{i}=g_{b a c}^{i}$ for all $i$, i.e. $\Gamma=\Gamma^{\prime}$.

Using (2.12) we can, starting at an arbitrary fermion field in each chain, arrange each one of the interaction Lagrangians such that all contracted fields stand next to each other (apart from the first and last field in a closed fermion chain). Denoting by $\hat{\chi}$ either $\chi$ or $\tilde{\chi}$, and by $\bar{\chi} \Gamma^{(\prime)} \hat{\chi}$ either $\bar{\chi} \Gamma \chi$ or $\bar{\chi} \Gamma^{\prime} \tilde{\chi}$, we obtain

$$
\begin{aligned}
& (-1)^{P} a\left(\overline{\widehat{\chi}} \Gamma^{(\prime)} \hat{\chi}\right) \ldots \ldots .\left(\overline{\widehat{\chi}} \Gamma^{(\prime)} \hat{\chi}\right)\left(\overline{\widehat{\chi}} \Gamma^{(\prime)} \hat{\chi}\right) a^{\dagger} \ldots \\
& \ldots\left(\overline{\bar{\chi}} \Gamma^{(\prime)} \hat{\chi}\right) \ldots \ldots(\underbrace{\bar{\chi}} \Gamma^{(\prime)} \hat{\chi})\left(\bar{\chi} \Gamma^{(\prime)} \hat{\chi}\right)
\end{aligned}
$$

where $a \overline{\bar{\chi}}$ stands for $b \bar{\chi}, b^{\dagger} \overline{\widetilde{\chi}}, d^{\dagger} \bar{\chi}$ or $d \overline{\tilde{\chi}}$ and $\widehat{\chi} a^{\dagger}$ for $\chi b^{\dagger}, \tilde{\chi} b, \chi d$ or $\tilde{\chi} d^{\dagger}$. Apart from the appearance of the charge-conjugate fields, (2.14) is of the same structure as in the case of Dirac fermions (2.4). The fermion number flow has been replaced by a fermion flow which corresponds to an orientation of each complete fermion chain. Again we only get signs from the permutation of the external creation and annihilation operators.

The contractions involving charge-conjugate fields are easily obtained from the ones of the original fields (2.6) and (2.7), and from the properties of the charge-conjugation

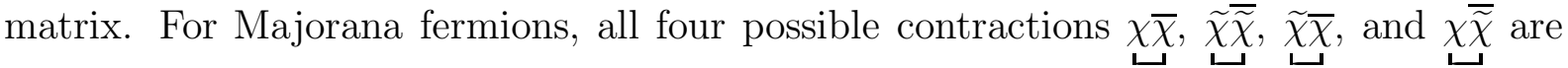
equal, for Dirac fermions only the two non-vanishing contractions $\chi \bar{\chi}$ and $\tilde{\chi} \bar{\chi}$ arise in (2.14). Using (2.10) the analytic expression for the propagator of the charge-conjugate fermion field becomes

$$
\begin{gathered}
\langle 0|T(\tilde{\chi} \overline{\bar{\chi}})| 0\rangle=C\left(\langle 0|T(\chi \bar{\chi})| 0\rangle T C^{-1}\right. \\
\longrightarrow \quad C S^{T}(p) C^{-1}=\frac{1}{-\not p-m}=S(-p)=: S^{\prime}(p),
\end{gathered}
$$

where the field $\tilde{\chi}$ is carrying momentum $p$, i.e. $\bar{\chi}$ carries momentum $-p$. Using (2.9) and the relations

$$
u(p, s)=C \bar{v}(p, s)^{T}, \quad v(p, s)=C \bar{u}(p, s)^{T},
$$

we get the explicit representation of the charge-conjugate fields from (2.5):

$$
\begin{aligned}
& \tilde{\chi}(x)=\int \frac{d^{3} k}{(2 \pi)^{3} 2 E} \sum_{s}\left[b^{\dagger}(k, s) v(k, s) e^{i k x}+d(k, s) u(k, s) e^{-i k x}\right] \\
& \overline{\widetilde{\chi}}(x)=\int \frac{d^{3} k}{(2 \pi)^{3} 2 E} \sum_{s}\left[b(k, s) \bar{v}(k, s) e^{-i k x}+d^{\dagger}(k, s) \bar{u}(k, s) e^{i k x}\right] .
\end{aligned}
$$

So the contractions between charge-conjugate fields and external operators in (2.14) yield

$$
\begin{aligned}
& \tilde{\chi} d_{i}^{\dagger}=\left\langle 0\left|\widetilde{\chi}(x) d^{\dagger}\left(p_{i}, s_{i}\right)\right| 0\right\rangle \quad \longrightarrow u\left(p_{i}, s_{i}\right) \\
& \stackrel{\vec{d}}{d_{i} \bar{\chi}}=\left\langle 0\left|d\left(p_{i}, s_{i}\right) \overline{\widetilde{\chi}}(x)\right| 0\right\rangle \quad \longrightarrow \quad \bar{u}\left(p_{i}, s_{i}\right) \\
& \overrightarrow{\widetilde{\chi}} b_{i}^{\dagger}=\left\langle 0\left|\overline{\tilde{\chi}}(x) b^{\dagger}\left(p_{i}, s_{i}\right)\right| 0\right\rangle \quad \longrightarrow \bar{v}\left(p_{i}, s_{i}\right) \\
& \stackrel{\stackrel{\sim}{b_{i}} \tilde{\chi}}{ }=\left\langle 0\left|b\left(p_{i}, s_{i}\right) \tilde{\chi}(x)\right| 0\right\rangle \quad \longrightarrow v\left(p_{i}, s_{i}\right) \text {. }
\end{aligned}
$$


As in the case of Dirac fermions we get a minus sign for every closed loop, for each

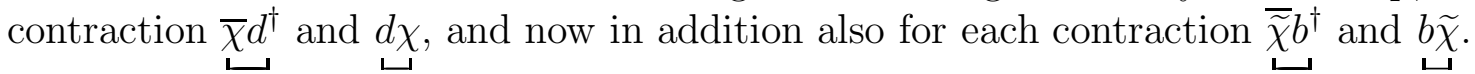

With these tools and the expressions for the couplings $\Gamma$ and their reversed counterparts $\Gamma^{\prime}$, the fermion chains are formed just as for Dirac fermions. Moreover the RSIF can be determined exactly as in the case of Dirac fermions, i.e.

(1) from the order of the external spinors,

(2) the number of fermion loops, and

(3) the number of $v$ 's and $\bar{v}$ 's.

Again the signs associated with the $v$ 's and $\bar{v}$ 's can be consistently omitted because (owing to our insistence on a fermion flow) different graphs contributing to the same process always differ by an even number of $v$ 's and $\bar{v}$ 's.

Two remarks are in place:

If, e.g. in (2.8), both $\chi$ 's are Majorana fermions, there are two equivalent non-vanishing Wick contractions

$$
\ldots(\bar{\chi} \Gamma \chi \underbrace{\chi}) . . . \quad \text { and } \quad \ldots .(\bar{\chi} \Gamma \chi) \ldots . .
$$

where in the first case we have to take the ordinary vertex $\Gamma$ and in the second the reversed one $\Gamma^{\prime}$. Since for Majorana fermions $\Gamma=\Gamma^{\prime}$, these two contractions yield the same result and cancel the factor $1 / 2$ in the corresponding interaction term in (2.1). This is exactly what happens for real scalar and vector fields.

Any of the fermion chains can be written down in the reverse way. For instance, for an open fermion chain with no external fermions and reversed vertices we obtain the following two equivalent representations

$$
\begin{gathered}
b_{1}(\bar{\chi} \Gamma \chi)(\bar{\chi} \Gamma \chi) \ldots \ldots(\bar{\chi} \Gamma \chi) b_{k}^{\dagger} \\
(-1) b_{k}^{b}\left(\overline{\tilde{\chi}} \Gamma^{\prime} \tilde{\chi}\right) \ldots \ldots\left(\overline{\tilde{\chi}} \Gamma^{\prime} \tilde{\chi}\right)\left(\overline{\tilde{\chi}} \Gamma^{\prime} \tilde{\chi}\right) b_{1}
\end{gathered}
$$

Of course both representations must yield the same analytic expression. To see this we insert our Feynman rules and arrive at:

$$
\begin{gathered}
\bar{u}\left(p_{1}, s_{1}\right) \Gamma S \Gamma \ldots \Gamma u\left(p_{k}, s_{k}\right) \\
(-1) \bar{v}\left(p_{k}, s_{k}\right) \Gamma^{\prime} \ldots \Gamma^{\prime} S^{\prime} \Gamma^{\prime} v\left(p_{1}, s_{1}\right) .
\end{gathered}
$$

Note that the factor $(-1)$ in $(2.20 \mathrm{~b})$ and $(2.21 \mathrm{~b})$ is due to the exchange of the external fermionic operators or spinors, respectively. Using (2.13), (2.15) and (2.16) we find

$$
\begin{aligned}
& -\bar{v}\left(p_{k}, s_{k}\right) \Gamma^{\prime} \ldots \Gamma^{\prime} S^{\prime} \Gamma^{\prime} v\left(p_{1}, s_{1}\right) \\
= & -v^{T}\left(p_{1}, s_{1}\right) \Gamma^{\prime T} S^{\prime T} \Gamma^{\prime T} \ldots \Gamma^{\prime T} \bar{v}^{T}\left(p_{k}, s_{k}\right) \\
= & -v^{T}\left(p_{1}, s_{1}\right) C^{-1} \Gamma S \Gamma \ldots \Gamma C \bar{v}^{T}\left(p_{k}, s_{k}\right) \\
= & \bar{u}\left(p_{1}, s_{1}\right) \Gamma S \Gamma \ldots \Gamma u\left(p_{k}, s_{k}\right)
\end{aligned}
$$

i.e. both representations - with fermion flows opposite to one another - do indeed yield identical results. This is of course true for arbitrary open and closed fermion chains, in particular also for conventional Dirac chains. 


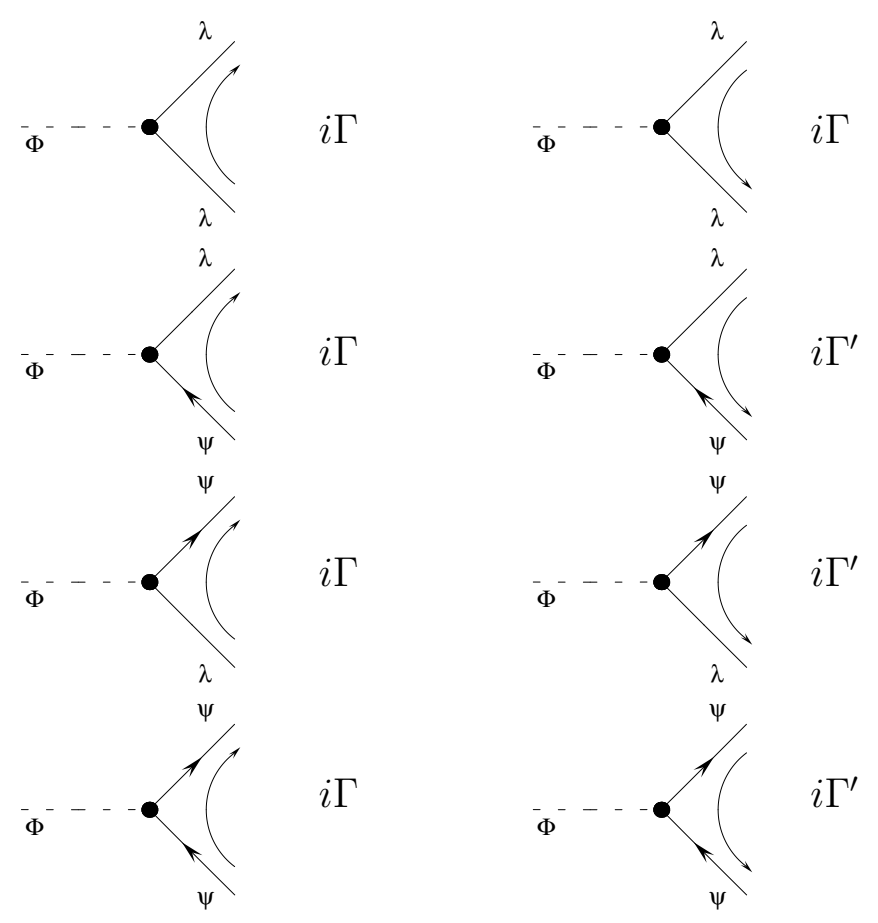

Figure 2.1: The Feynman rules for fermionic vertices with orientation (thin arrows)

\subsection{Formulation of the Feynman rules}

Now we can summarize our Feynman rules:

Fermions are denoted by solid lines. For Dirac fermions, each line carries an arrow which indicates the fermion number flow. Majorana lines do not carry arrows.

The fermionic vertices are read off from the Lagrangian as usual, but for every vertex containing Dirac fermions we need two expressions, the direct one ( $\Gamma$ from $\bar{\chi} \Gamma \chi)$ and the reversed one $\left(\Gamma^{\prime}\right.$ from $\bar{\chi} \Gamma^{\prime} \widetilde{\chi}$ ) (see Fig. 2.1). For a pure Majorana fermion vertex there is only one expression because $\Gamma=\Gamma^{\prime}$.

For Dirac fermions there exists the usual propagator $S(p)$ and the reversed one $S^{\prime}(p)=$ $S(-p)$ as well the usual spinors and their reversed counterparts. For Majorana fermions there are only the usual propagator and spinors and no reversed ones (see Figs. 2.2 and 2.3). Note that with respect to the fermion flow the reversed propagator and spinors equal the usual ones and there is only one propagator and the usual spinors ${ }^{3}$.

From these ingredients the Feynman amplitudes are obtained as follows:

- Draw all possible Feynman diagrams for a given process.

- Fix an arbitrary orientation (fermion flow) for each fermion chain.

\footnotetext{
${ }^{3}$ The usual Feynman rules for propagators and external fermions depend on the relative orientation of fermion number flow and momentum. Our Feynman rules are obtained from the usual ones when substituting the fermion number flow by the fermion flow using the same set of analytical expressions.
} 


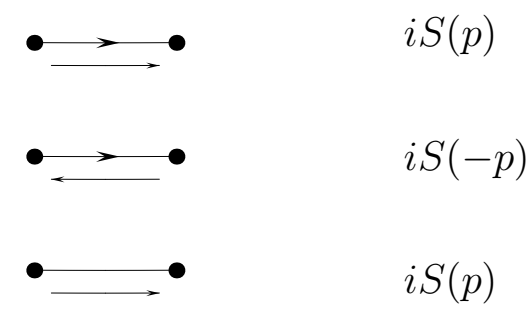

Figure 2.2: The Feynman rules for fermion propagators with orientation (thin arrows). The momentum $p$ flows from left to right.

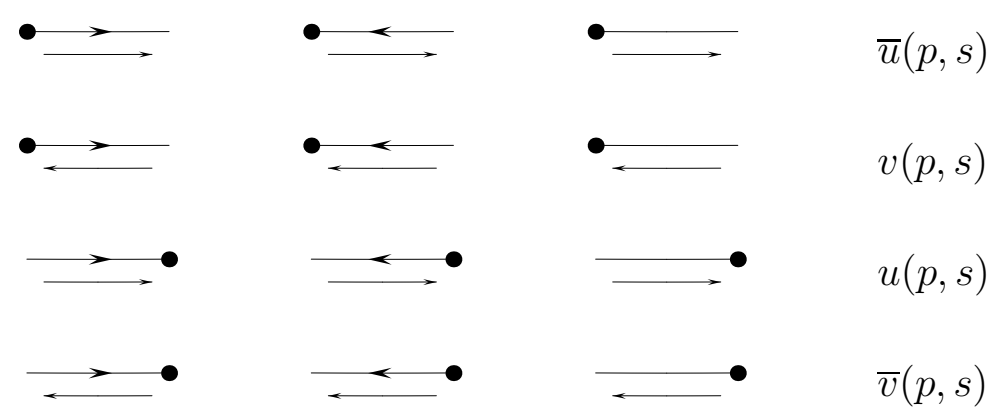

Figure 2.3: The Feynman rules for external fermion lines with orientation (thin arrows). The momentum $p$ flows from left to right.

- Start at an external leg (for closed loops at some arbitrary propagator) and write down the Dirac matrices proceeding opposite to the chosen orientation (fermion flow) through the chain.

- For each internal propagator, external line and vertex insert the appropriate analytic expression as given in Figs. 2.1, 2.2 and 2.3 corresponding to the chosen fermion flow. This implies that if the orientation is opposite to the flow of fermion number the reversed vertices $\Gamma^{\prime}$, propagators $S(-p)$ and spinors have to be taken.

- Multiply by a factor (-1) for every closed loop.

- Multiply by the permutation parity of the spinors in the obtained analytical expression with respect to some reference order.

- As far as the determination of the combinatorial factor is concerned, Majorana fermions behave exactly like real scalar or vector fields. 


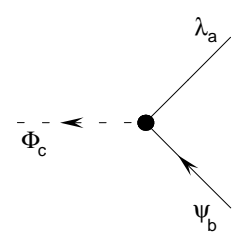

a)

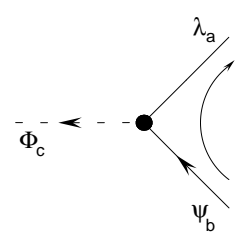

b)

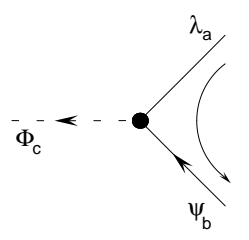

c)

Figure 3.1: Feynman diagram for $\Phi \rightarrow \lambda \psi$

\section{$3 \quad$ Examples}

We illustrate the application and universality of our Feynman rules by giving some examples. In order to allow an easy comparison, we discuss the same processes as in earlier papers on Majorana Feynman rules [1]-[4].

\section{$3.1 \Phi \rightarrow \lambda \psi$}

We start out with the simple decay of a scalar $\Phi$ into a Dirac and a Majorana fermion $\Phi \rightarrow \lambda \psi$, which already suffers from sign ambiguities in the conventional treatment $[1,2]$. In our formulation the Feynman graph in Fig. 3.1a can be read in two different ways, as indicated in Fig. 3.1b and Fig. 3.1c. From Fig. 3.1b we find

$$
i \mathcal{M}=i \bar{u}_{a} \Gamma_{i} v_{b} k_{a b c}^{i},
$$

where the reference order of the external fermions has been chosen as $(a, b)$. The opposite orientation (Fig. 3.1c) yields

$$
i \mathcal{M}^{\prime}=(-1) i \bar{u}_{b} \Gamma_{i}^{\prime} v_{a} k_{a b c}^{i}
$$

where the explicit $(-1)$ comes from the permutation parity of the order $(b, a)$ with respect to the reference order $(a, b)$. The equivalence of (3.1a) and (3.1b) can be easily shown using (2.16) and (2.13)

$$
i \mathcal{M}^{\prime}=-i v_{a}^{T}\left(\Gamma_{i}^{\prime}\right)^{T} \bar{u}_{b}^{T} k_{a b c}^{i}=i \bar{u}_{a} \Gamma_{i} v_{b} k_{a b c}^{i}=i \mathcal{M} .
$$

The authors of Ref. [3] do not deduce the extra ( -1$)$ from the exchange of the fermion spinors but include it into their definition of the vertices $\Gamma^{\prime}$. Obviously both methods lead to equivalent results for the simple vertex diagram of Fig. 3.1a. However, for more complicated processes their definition of the vertices cannot resolve all sign ambiguities. Even worse it obscures the graphic determination of the RSIF.

\section{$3.2 \psi \psi \psi \rightarrow \Phi \Phi$}

To show this we consider the process depicted in Fig. 3.2. Using the Feynman rules of 


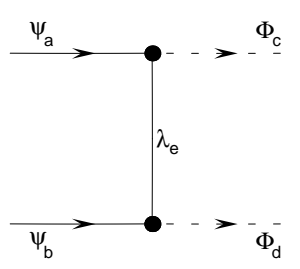

a)

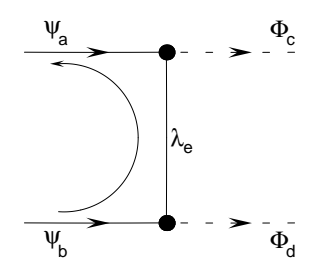

b)

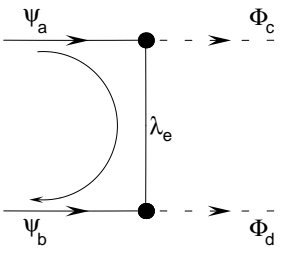

c)

Figure 3.2: Feynman diagram contributing to $\psi \psi \rightarrow \Phi \Phi$

Ref. [3] the result depends on the chosen fermion flow. Because the same vertices appear for both orientations the sign ambiguity cannot be resolved by redefining vertices.

In our formulation the sign is determined from the permutation parity of the spinors with respect to a reference order as in the preceeding example ${ }^{4}$. Fixing the reference order as $(a, b)$, we find for the fermion flow indicated in Fig. $3.2 \mathrm{~b}$

$$
i \mathcal{M}=-i \bar{v}_{a} \Gamma_{i}^{\prime} S\left(p_{c}-p_{a}\right) \Gamma_{j} u_{b} k_{e a c}^{i} k_{e b d}^{j}
$$

and for the reversed fermion flow (Fig. 3.2c)

$$
i \mathcal{M}^{\prime}=(-1)(-i) \bar{v}_{b} \Gamma_{j}^{\prime} S\left(p_{a}-p_{c}\right) \Gamma_{i} u_{a} k_{e a c}^{i} k_{e b d}^{j}=i \mathcal{M}
$$

This example demonstrates that in contrast with the Feynman rules in Refs. [1, 2, 3] our rules are independent of the orientation of the internal Majorana lines. In fact we do not even introduce an orientation of those lines but an orientation of whole fermion chains. Evidently our rules are independent of the direction of internal fermion momenta. We note that all sign ambiguities which were left in Ref. [3] are due to the definition of the reversed vertices given there and are naturally, resolved using our definition.

\section{$3.3 \psi \psi \rightarrow \Phi \psi \lambda$}

In order to illustrate that the RSIF emerges naturally, we consider the diagrams in Fig. $3.3^{5}$. The corresponding analytical expressions read

$$
\begin{aligned}
& i \mathcal{M}_{1}=(+1) i \bar{v}_{a} \Gamma_{i}^{\prime} S\left(p_{c}-p_{a}\right) \Gamma_{j} u_{b} D\left(p_{f}+p_{g}\right) \bar{u}_{f} \Gamma_{k} v_{g} k_{e a c}^{i} k_{e b d}^{j} k_{g f d}^{k *} \\
& i \mathcal{M}_{2}=(-1) i \bar{u}_{f} \Gamma_{k} S\left(p_{a}-p_{c}\right) \Gamma_{i} u_{a} D\left(p_{b}-p_{g}\right) \bar{u}_{g} \Gamma_{j} u_{b} k_{e a c}^{i} k_{g b d}^{j} k_{e f d}^{k *} \\
& i \mathcal{M}_{3}=(+1) i \bar{u}_{f} \Gamma_{k} S\left(p_{b}-p_{c}\right) \Gamma_{j} u_{b} D\left(p_{a}-p_{g}\right) \bar{u}_{g} \Gamma_{i} u_{a} k_{g a d}^{i} k_{e b c}^{j} k_{e f d}^{k *},
\end{aligned}
$$

where the explicit signs $( \pm 1)$ result from the order of the fermions $(a, b, f, g),(f, a, g, b)$, $(f, b, g, a)$ of the chosen representation of the diagrams, with respect to the reference order

\footnotetext{
${ }^{4}$ Note that this cannot be done consistently for both processes in Fig. 3.1 and Fig. 3.2 if the vertices are defined as in Ref. [3].

${ }^{5}$ This is not the complete set of diagrams contributing to $\psi \psi \rightarrow \Phi \psi \lambda$.
} 


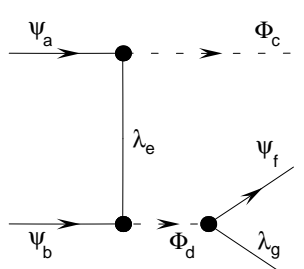

1)

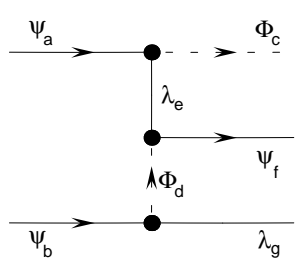

2)

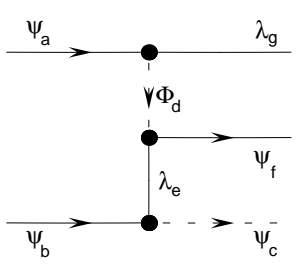

$3)$

Figure 3.3: Feynman diagrams contributing to $\psi \psi \rightarrow \Phi \psi \lambda$

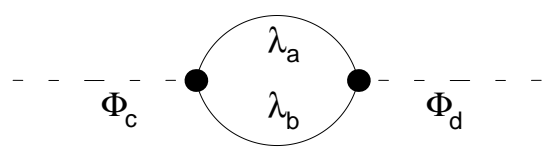

Figure 3.4: Feynman diagram containing a Majorana fermion loop

$(a, b, f, g)$, in agreement with the Wick theorem. The orientation of any fermion chain may be reversed without changing the result, e.g. for the diagram of Fig. 3.3c we have in addition the following three equivalent representations

$$
\begin{aligned}
& i \mathcal{M}_{3}=(-1) i \bar{u}_{f} \Gamma_{k} S\left(p_{b}-p_{c}\right) \Gamma_{j} u_{b} D\left(p_{a}-p_{g}\right) \bar{v}_{a} \Gamma_{i}^{\prime} v_{g} k_{g a d}^{i} k_{e b c}^{j} k_{e f d}^{k *} \\
& i \mathcal{M}_{3}=(+1) i \bar{v}_{b} \Gamma_{j}^{\prime} S\left(p_{c}-p_{b}\right) \Gamma_{k}^{\prime} v_{f} D\left(p_{a}-p_{g}\right) \bar{v}_{a} \Gamma_{i}^{\prime} v_{g} k_{g a d}^{i} k_{e b c}^{j} k_{e f d}^{k *} \\
& i \mathcal{M}_{3}=(-1) i \bar{v}_{b} \Gamma_{j}^{\prime} S\left(p_{c}-p_{b}\right) \Gamma_{k}^{\prime} v_{f} D\left(p_{a}-p_{g}\right) \bar{u}_{g} \Gamma_{i} u_{a} k_{g a d}^{i} k_{e b c}^{j} k_{e f d}^{k *} .
\end{aligned}
$$

\subsection{Loop diagrams}

Our Feynman rules given in section 2.4 obviously apply to any order of perturbation theory. We illustrate this by giving the analytical expression for the diagram in Fig. 3.4:

$$
\begin{aligned}
i \mathcal{M} & =(-1) \frac{1}{2} \int \frac{d^{4} q}{(2 \pi)^{4}} \operatorname{Tr}\left\{\Gamma_{i} S(k+q) \Gamma_{j} S(q)\right\} g_{a b c}^{i} g_{b a d}^{j} \\
& =(-1) \frac{1}{2} \int \frac{d^{4} q}{(2 \pi)^{4}} \operatorname{Tr}\left\{\Gamma_{j} S(-k-q) \Gamma_{i} S(-q)\right\} g_{b a c}^{i} g_{a b d}^{j}
\end{aligned}
$$

which is independent of the chosen fermion flow. The explicit factor $(-1)$ originates from the fermion loop. The factor $1 / 2$ is the usual combinatorial factor for identical particles. Note that a combinatorial factor associated with fermions only appears for a loop containing exactly two identical Majorana fermions. 


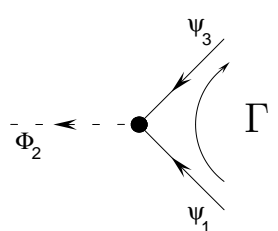

a)

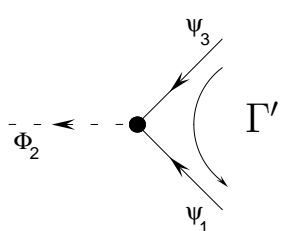

b)

Figure 4.1: Vertex with two clashing Dirac fermions

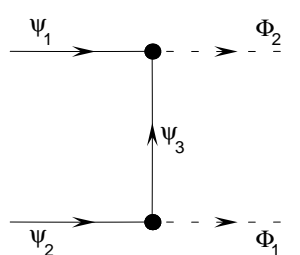

a)

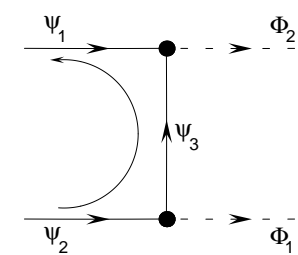

b)

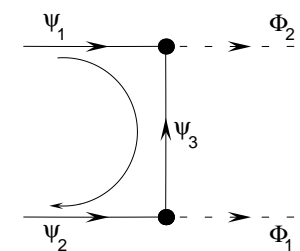

c)

Figure 4.2: Feynman diagram contributing to $\psi_{1} \psi_{2} \rightarrow \Phi_{1} \Phi_{2}$

\section{Lagrangians with explicit charge-conjugate fermion fields}

In general Lagrangians - in particular in the supersymmetric standard model — there appear explicit charge-conjugate fields. As an example we consider

$$
\mathcal{L}=\ldots+\bar{\psi}_{1} \Gamma_{i} \psi_{2} \Phi_{3}^{*} h_{123}^{i}+\bar{\psi}_{3} \Gamma_{i} \psi_{2} \Phi_{1}^{*} h_{321}^{i}+\widetilde{\widetilde{\psi}_{3}} \Gamma_{i} \psi_{1} \Phi_{2}^{*} h_{312}^{i} .
$$

Note that the charge-conjugate field $\overline{\widetilde{\psi}}$ cannot be eliminated via field redefinitions such as $\psi_{3} \rightarrow \widetilde{\psi}_{3}$ without introducing $\widetilde{\psi}_{2}$ or $\widetilde{\psi}_{1}$. In the conventional treatment, these fields are expressed in terms of the original fields and the $C$ matrix using (2.9). This leads to the explicit appearance of the charge-conjugation matrix in the Feynman rules and to difficulties in the determination of the RSIF directly from the graphs.

On the other hand interactions with explicit charge-conjugate fields fit naturally in our Feynman rules which in fact introduce $\widetilde{\psi}$. As in section 2 we assign two different analytical expressions to the vertex derived from the last term in (4.1)

$$
\widetilde{\widetilde{\psi}_{3}} \Gamma_{i} \psi_{1} \Phi_{2}^{*} h_{312}^{i}=\overline{\widetilde{\psi}_{1}} \Gamma_{i}^{\prime} \psi_{3} \Phi_{2}^{*} h_{312}^{i},
$$

each one corresponding to a definite fermion flow as shown in Fig. 4.1a and Fig. 4.1b.

As an example consider the contribution to the process $\psi_{1} \psi_{2} \rightarrow \Phi_{1} \Phi_{2}$ depicted in Fig. 4.2a. Assuming reference order $(1,2)$ we obtain for Fig. 4.2b (using the vertex of Fig. 4.1b)

$$
i \mathcal{M}=-i \bar{v}_{1} \Gamma_{i}^{\prime} S\left(p_{2}-p_{1}\right) \Gamma_{j} u_{2} h_{312}^{i} h_{321}^{j}
$$

and for the opposite fermion flow Fig. 4.2c (using Fig. 4.1a)

$$
i \mathcal{M}^{\prime}=(-1)(-i) \bar{v}_{2} \Gamma_{j}^{\prime} S\left(p_{1}-p_{2}\right) \Gamma_{i} u_{1} h_{312}^{i} h_{321}^{j} .
$$

Equations (4.3a) and (4.3b) are equivalent and formally the same as (3.3a) and (3.3b). 


\section{Conclusion}

We have given simple algorithmic Feynman rules for Majorana fermions and arbitrary fermion-number-violating interactions resembling closely those for Dirac fermions. They are based on a well-defined fermion flow, involve only the usual Dirac propagator and vertices without explicit charge-conjugation matrices. In our formulation of the Feynman rules all relative sign ambiguities are resolved and the relative sign of interfering Feynman diagrams is determined exactly as in the case of Dirac fermions.

Our rules facilitate practical calculations considerably and can easily be implemented into symbolic Feynman diagram generators.

\section{References}

[1] S.K. Jones and C.H. Llewellyn Smith, Nucl. Phys. B217 (1983) 145.

[2] H.E. Haber and G.I. Kane, Phys. Rep. 117 (1985) 76.

[3] E.I. Gates and K.L. Kowalski, Phys. Rev. D37 (1988) 938.

[4] J. Gluza and M. Zrałek, Phys. Rev. D45 (1992) 1693.

[5] J. Küblbeck, M. Böhm and A. Denner, Comp. Phys. Comm. 60 (1990) 165,

H. Eck and J. Küblbeck, Guide to FeynArts 1.0, University of Würzburg, 1992.

[6] C. Itzykson and J. Zuber, Quantum field theory, McGraw-Hill (1980). 